\title{
Tattiche testuali
}

\section{Roberto Fedi}

Notevole fortuna sembra avere, in questi ultimi tempi, la discussione sulle difficoltà, l'uso, le tecniche della scrittura, creativa e anche saggistica (per quest'ultima: Marius 28-31). Credo sia opportuno estendere la questione alle difficoltà (o almeno ai problemi) della lettura, e della susseguente interpretazione testuale: e mi riferisco qui anche alla dimensione più quotidiana dell'insegnamento della letteratura. Come si vedrà è un argomento che è fatalmente destinato a sconfinare dalle più semplici pratiche testuali a quelle, assai più laboriose e complicate, dell'interpretazione vera e propria di un testo-un tema che oggi sarebbe bene affrontare con franchezza, credo, dopo anni caratterizzati vicendevolmente dai rigori delle codificazioni semiologiche o strutturali, e viceversa dalle quasi illimitate franchigie delle teorie decostruzioniste. Mi sia concesso di ritenere, per altro, non del tutto casuale il fatto che la sollecitazione-se qualcuno vorrà raccoglierla - venga da chi ha soprattutto esperienza di insegnamento in ambito europeo, pur non essendo privo di contatti e di pratica diretta in Nord America e negli Stati Uniti in particolare. Mi limiterò ad accenni, e a qualche esempio.

La prima cosa che colpisce colui che, venendo da abitudini critiche e di insegnamento diverse (italiane nel mio caso) si trovi ad insegnare letteratura negli Stati Uniti o in Canada, è quella che chiamerei la 'posizione' del lettore-studente, il suo punto di vista o focus. È, per molti aspetti, diametralmente opposta a quella del suo omologo d'oltre Atlantico: quest'ultimo è infatti portato, per lunga consuetudine che inizia almeno da quelle che in Italia si chiamano Scuole Medie, a considerare il testo letterario nella sua articolazione storica e, potremmo dire, intertestuale. Il testo insomma, qualsiasi testo, deve subire prima della esegesi personale da parte del fruitore un lungo lavoro di identificazione: storica, filologica, di fonti. È inserito in una costellazione di altri testi (dello stesso autore o di altri contemporanei) di cui entra a fare parte stabilmente; ne viene analizzata l'appartenenza ad un 'genere' (la poesia lirica, la poesia epica, la novellistica, ecc.); se ne chiarisce l'aspetto filologico anche in senso evolutivo (le diverse stesure, le eventuali varianti, la storia del testo); viene letto, infine, come parte di un tutto che può essere costituito dalla ideologia poetica del suo autore (spesso scandita nel suo sviluppo), e anche dalla 'famiglia' testuale a cui appartiene, o dal 'sistema' in cui si inscrive (la poesia petrarchista del secolo XVI, il romanzo storico dell'Ottocento, ecc.). In definitiva, si storicizza (o si cerca: mi riferisco qui ovviamente alle intenzioni operative): secondo un costume ormai consolidato che, in Italia almeno, risale all'incirca alla metà del secolo scorso, ed al prevalere anche 
in senso didattico delle teorie e delle idee romantiche, corroborate in seguito dallo Storicismo positivista (Ossola 95-6). Si aggiunga a ciò il fatto che in Italia i programmi di insegnamento nelle Scuole - Università compresa, sia pure con varie concessioni all'autonomia - sono unificati e stabiliti in via legislativa.

È questo un metodo di lettura (che però rinvia direttamente ad atteggiamenti critici) radicalmente diverso da quello comunemente in uso nelle aule universitarie nordamericane. Se il lettore europeo si pone di fronte al testo sempre presupponendo l'esistenza ramificata di un 'sistema', il suo collega d'oltre Atlantico percepisce solitamente il testo piuttosto nella sua singolarità - se non vado errato. Da qui, credo, la straordinaria libertà che quasi sempre è riservata all'esegeta, il quale viene portato ad interrogarsi su cosa significhi (o voglia adombrare per simboli) quel testo in sé, e non in quanto portatore di informazioni più generali - sul suo autore, sul suo secolo, sul 'genere' letterario a cui si apparenta, ecc. La codificazione tecnica (in particolare delle scuole semiologiche o strutturali) ha appena scalfito, supportandola con strumentazioni e definizioni meno generiche e personalistiche, quella fondamentale libertà di essere per così dire soli con il testo, quel rapporto a due fra esegeta e parola scritta che rimane - o così almeno mi sembra - forse la più suggestiva delle abitudini critiche o di studio americane (che ciò rinvii, magari alla lontana, anche alla tradizione protestante di auto-interpretazione della Bibbia, così diversa dalla subordinazione all'ufficialità ecclesiastica del mondo cattolico, è ipotesi che nasce spontanea, ma che non sono in grado di verificare).

Non credo che questa netta differenza di modalità critiche ed esegetiche derivi solo dal leggero peso di una più recente tradizione, di contro all'onda lunga della storiografia europea. La storia letteraria intesa come 'manuale', e come 'discorso' dotato di propria vita e di autonoma dignità quasi in opposizione alla lettura diretta, che comunque viene sempre in subordine, è-come già si è osservato - un'eredità talvolta molto pesante del Romanticismo europeo: fino alla metà del secolo scorso il modello operante e imperante era al contrario, anche in Italia, quello della lettura e quindi della scrittura come 'imitazione', dell'apprendimento della lingua e delle tecniche come esercizio retorico che potremmo chiamare anche noi "intransitivo" (Genette 23-42), in altre parole non finalizzato ad altro che alla "ripetizione" del testo specialmente poetico - da qui infatti l'uso imperante dei trattati di retorica, e la larga diffusione delle Cattedre di eloquenza. In questo panorama, potremmo aggiungere, lo scrittore assumeva quindi una funzione essenzialmente apedagogica, attenta alla committenza, lontana - almeno programmaticamente - da quello che dall'Illuminismo e dalla Rivoluzione Francese in poi si è soliti definire l'engagement dell'intellettuale, in sostanza la sua dimensione educativa e, spesso, anticlassicistica (Battistini e Raimondi 182 sgg.). È un passaggio fondamentale quello che si verifica alla metà del secolo scorso fra la letteratura - ed il testo letterario-come modello appunto di eloquenza, e la stessa 
come "modello di consapevolezza": di questo transito, Francesco De Sanctis è in Italia l'interprete più autorevole, ed a sua volta il modello esemplare ne è la sua Storia delle letteratura italiana (Dionisotti 12-5; 27-30).

Ne è derivata l'idea, ancora vivissima, della funzione della letteratura (nella sua accezione di 'testo' letterario) come parte inscindibile di un 'sistema' che ha, al suo vertice, la storiografia letteraria come elemento unificante e finalizzato, e l'intellettuale come suo profeta autorizzato; il tutto, concorrente alla costituzione di un modello unificatore, valido nel tempo e strutturalmente solido. La letteratura, insomma, ha continuato a devolvere una sua eredità formativa, sicuramente importante in una nazione (sto ancora parlando dell'Italia) giunta alla sua unificazione solo nella seconda metà del secolo XIX. Non a caso, in questo panorama, minore importanza assumevano fino a pochi anni fa le voci 'eccentriche': gli scrittori dialettali o anonimi, le opere composte in lingue diverse da quel modello 'alto' proposto come esempio, gli intellettuali italiani che operavano all'estero, o quelli che scrivevano per suggestioni di letterature diverse da quella nazionale - che, infatti, sono un acquisto recente e largamente innovativo della critica più avvertita. Accanto a queste voci più emarginate, anche la letteratura contemporanea e Novecentesca subiva un processo di accantonamento (almeno nel settore scolastico e universitario), in quanto non ancora portatrice di tradizione, e spesso a sua volta compromessa con altre intertestualità (la musica, le arti figurative, le lingue straniere). Negli ultimi anni si è assistito ad un trend opposto, secondo il quale lo studente-lettore, reagendo all'eccessivo storicismo di matrice idealista che quasi riduceva il testo in una sorta di gabbia interpretativa, ha sempre più teso ad attualizzare nei testi un suo bisogno effettivo di concretezza, una sua attrazione forte verso il presente nel quale riconoscersi. II testo, così, è divenuto come suol dirsi un 'pretesto': le più attuali tattiche di lettura, e una forte carica ideologica o almeno ideologizzante (la psicanalisi, il marxismo, la semiologia), hanno funzionato egregiamente, per un breve periodo, in questa agitata rincorsa al primato della tematica (il significato a spese del significante), e in questa spinta alla generalizzazione (il bisogno di riconoscersi nell'hic et nunc e nella massa) ma al tempo stesso alla personalizzazione del processo cognitivo (l'io del lettore che reagisce di fronte al testo). Direi che ne sono scaturite due tendenze, entrambe moriture: il tecnicismo assolutizzante della nuova esegesi (la 'misurazione' del testo, la riduzione della pagina scritta a formula ed a una serie di funzioni, indipendentemente dalla sua natura artistica); e la rinascita, sotto forme naturalmente molto più ingenue, di un estetismo generico e sbarazzino secondo il quale è 'bello' ciò in cui ci si riconosce o si ritrova il proprio 'vissuto' - o ciò che si crede che esso sia, secondo gli standards di moda ("trovo bella la poesia X perché mi ricorda ..." ", "non mi piace il racconto Y perché irreale ..." ", ecc.: Ossola 105). Una rivincita, ahimè molto malridotta, del più banalizzato Croce.

Credo che in Nord America le cose siano andate molto diversamente, ma che vi si possa riscontrare oggi una condizione di incertezza non dissimile da 
quella che si vive in Europa. Non a caso è sostanzialmente nelle Università americane che è nata la Scuola del Decostruzionismo, verso la quale il più recente volume di Umberto Eco appunta più d'una riserva (I limiti dell'interpretazione), come lo stesso autore ha tenuto a ribadire in un'intervista di qualche mese fa ("Intervista"), opponendo all'uso del testo come materiale di esercitazione o campo di riconoscimento delle proprie pulsioni un'idea più "oggettiva" dell'esegesi testuale. Né per altro era casuale il fatto che, in Opera aperta, l'Eco del 1962 si giovasse soprattutto di pezze d'appoggio extra-europee per l'esposizione del concetto di testo come "multiformità di significati”, disponibile alla libera reazione del fruitore (37-42). D'altra parte, almeno in questa fase della lettura e dell'interpretazione, non so quanto possa essere utile il concetto (risalente a Frye) della critica come elaboratore di una struttura sistematica del sapere, svincolata da ogni considerazione tradizionale di valore (Frye; Kermode). Ci troviamo, insomma, in una scomoda posizione, ad un bivio: da una parte l'idea ottocentesca e idealistica di una lettura come riconoscimento dei dati 'poetici' di una serie di Autori molto rappresentativi, sintetizzati in un 'discorso' che tiene in primo piano quasi sempre le loro 'poetiche'; dall'altra la ricerca di informazioni sul testo, decodificato nei suoi meccanismi formali e interrogato a partire dalla sua natura di prodotto linguistico (lo strutturalismo) o semico (la semiologia). È probabilmente osservando questa frattura storica, nella quale stiamo vivendo, che al di qua o al di là dell'Atlantico vale la pena di interrogarsi. Cos'è, insomma, il testo? e, vorrei aggiungere: cosa significa, oggi, la lettura del testo in un ámbito magari di apprendistato (l'Università, la scuola), ma che non può prescindere dalle tecniche 'alte' della ricerca scientifica, e anzi spesso ne è condizionata quasi inconsapevolmente? Una volta data per scontata l'illiceità della più disperante parafrasi (quella secondo cui non c'è differenza fra "Sempre caro mi fu quest'ermo colle" e "Questa collinetta mi è sempre piaciuta", insomma), nonché della lettura esclamativa, da guida turistica semi-acculturata (nel suo grado più alto, il cosiddetto "piacere" della lettura), non credo che si possa, comunque, 'approfittarsi' del testo, poetico o narrativo, ai fini di un esercizio (alto o modesto a seconda dei casi) della propria capacità di reazione: se ciò giova spesso all'analisi delle minime strutture formali o dei grumi di significato latente, ci sfuggirà poi sempre la sintesi pur necessaria alla definizione di un'informazione generale sull'opera (lo stesso Eco ha detto più volte, non sempre scherzando, che molte delle supposizioni critiche intorno ai suoi romanzi lo hanno colto francamente di sorpresa: "Intervista"). Né, d'altra parte, si può accettare in toto il principio di una scrittura inconscia, secondo la quale solo il lettore, partecipe alla pari dello scrittore, può decodificare il non detto: tecnica da utilizzare con cautela e su cui giova riflettere, per evitare il grottesco di interpretazioni anche spericolate frutto di banali errori di lettura o, addirittura, di stampa (per un caso risolto, relativo a Eugenio Montale, cfr. Rebay 33-53; Graziosi 303). Nel testo confluiscono, o almeno a me sembra che confluiscano, spinte diverse, suggestioni eterogenee, memorie ed 
echi che si intrecciano nella organizzazione della sua struttura e si condensano nelle singole unità; $e$ sono queste informazioni che siamo chiamati a riconoscere. Ed è evidente inoltre che, intendendo la storia letteraria come un intersecarsi di testi più che come una successione di autori, come Archivio aperto della memoria più che come Museo, non possiamo non tenere conto del making del testo nella sua stratificazione nel tempo: nella sua dinamica anche filologica (Caretti), e non solo nella polisemia dei significati, simbolici o meno. Superando la storicizzazione anche di scuola marxista che era portata ad intendere il testo come 'documento', quindi essenzialmente utile per ricostruzioni che gli rimanevano per larga parte eterogenee, irrelate come erano con la sua natura di messaggio unitario, dovremo porci piuttosto il problema della "storicità dei codici" - per dirla con Segre: in altre parole della distinzione - ma della possibile omogeneizzazione e interazione all'atto della lettura - dei codici storicamente accertati dell'emittente (lo scrittore, all'atto della sua produzione letteraria, nel suo contesto) e del ricevente (il lettore, che ricorre ai codici di cui dispone per interpretare il testo). Con il che si vede come, dietro quello dell'interpretazione, si imponga sempre di più il basilare problema dell'incontro fra due culture: che si ripropone sempre, con le sue anche illuminanti possibilità, ad ogni nostro atto della lettura, anche nella sua più quotidiana accezione scolastica (Segre 118-9).

Fatto centrale rimane comunque, nella identificazione dei messaggi di un testo, e nella sua interpretazione in sede anche di lettura, il riconoscimento della sua sostanziale natura di oggetto retorico, fatto di elementi di significato e perciò-almeno altrettanto-artisticamente elaborato; e comunque irriconoscibile se di esso si voglia cogliere solo il primo livello interpretativo, quello della 'realtà', e quindi sostanzialmente della parafrasi (Graziosi 302-3). Un'elaborazione formale, quella del testo, che dovremo riconoscere nella sua possibilità di essere sempre ricondotta ad una 'storia' stilistica, ad un making testuale e naturalmente, in certi casi, anche più ampiamente compositivo e strutturale (si pensi all'organizzazione dei canzonieri lirici, ed alla loro unità nella molteplicità; in altre parole, al loro essere latori di messaggi il cui senso non deriva dalla mera sommatoria dei loro fragmenta: Fedi, Gorni, Santagata), in uno svolgimento anche diacronico ricco il più possibile di riferimenti intertestuali, tendenti ad organizzarsi in un 'sistema', insomma in una selva di echi che va naturalmente districata, ma non ridotta a giardino artificiale o peggio ancora abbattuta.

Per il resto, ai più ingenui studenti-lettori che cadessero nel primo e più fatale errore (quello di ritenere un testo, anche narrativo, essenzialmente 'reale', e perciò disponibile alla parafrasi ed alle suggestioni individuali e pre-critiche), si può sempre ricordare l'illuminante aneddoto su Henri Matisse. Avendo costui mostrato certi suoi quadri di figure femminili ad un'amica, si sentì obiettare che le donne non avevano certo quell'aspetto. "Ma questa non è una donna" rispose il pittore. "È un quadro".

Università di Salerno 


\section{OPERE CITATE}

Battistini, Andrea e Ezio Raimondi. "Retoriche e poetiche dominanti". Letteratura italiana. Le forme del testo. Vol. 3.1. Teoria e poesia. Ed. Alberto Asor Rosa. Torino: Einaudi, 1984: 5-339.

Caretti, Lanfranco. "Filologia e critica". In Antichi e moderni. Torino: Einaudi, 1976: 471-88.

[11 saggio era apparso nel 1952.]

Croce, Benedetto. Estetica come scienza dell'espressione e linguistica generale. Teoria e storia.

Palermo: Sandron, 1902.

. Problemi di estetica e contributi alla storia dell'estetica italiana. Bari: Laterza,

1910.

Nuovi saggi di estetica. Bari: Laterza, 1920.

De Sanctis, Francesco. Storia della letteratura italiana. Napoli: Morano, 1870-71.

Dionisotti, Carlo. Geografia e storia della letteratura italiana. Torino: Einaudi, 1967.

Eco, Umberto. Opera aperta. Milano: Bompiani, 1962. Rpt. 1967, 1976.

I limiti dell'interpretazione. Milano: Bompiani, 1990.

Intervista con Laura Lilli. La Repubblica 7 Dic. (1990).

Fedi, Roberto. La memoria della poesia. Canzonieri, lirici e libri di rime nel Rinascimento. Roma: Salerno Ed., 1990.

Frye, Northrop. Anatomy of Criticism. Princeton: Princeton UP, 1957.

Genette, Gérard. "Rhétorique et inseignement". Figures II. Paris: Seuil, 1969: 23-42.

Gorni, Guglielmo. "Le forme primarie del testo poetico". Letteratura italiana. Le forme del testo. Vol. 3.1. Teoria e poesia. Ed. Alberto Asor Rosa. Torino: Einaudi, 1984: 504-18.

Graziosi, Elisabetta. "Come si gioca con un testo letterario". Come si legge un testo. Da Dante a Montale. Ed. Maria Luisa Altieri Biagi. Milano: Mursia, 1989: 299-323.

Kermode, Frank. "Chiedetelo a Shakespeare”. La Repubblica 8 Dic. (1990).

Marius, Richard. “On Academic Discourse”. Profession 90 (1990): 28-31.

Ossola, Carlo. "Testo poetico e storia letteraria". Didattica dell'italiano. Ed. Mario Ricciardi. Torino: Edizione Stampatori, 1976: 94-122.

Rebay, Luciano. "1 diáspori di Montale". Italica 46.1 (1969): 33-53.

Santagata, Marco. Dal sonetto al canzoniere. Ricerche sulla preistoria e la costituzione di un genere. Padova: Liviana, 1989 (1.a ed. 1979).

Segre, Cesare. "Testo letterario, interpretazione, storia: linee concettuali e categorie critiche". Letteratura italiana. L'interpretazione. Vol. 4. Ed. Alberto Asor Rosa. Torino: Einaudi, 1985: 21-140. 